\title{
A Single-sensor High-resolution Panoramic Optical Mapping Configuration for Simultaneous Non-overlapped Complete Atrial and Ventricular Parametric Imaging
}

\author{
Conrado J. Calvo ${ }^{1,2,5}$, Antonio Guill ${ }^{1,5}$, Alvaro Tormos ${ }^{1,5}$, Luis Such-Miquel ${ }^{4}$, Manuel Zarzoso ${ }^{4}$, \\ Oscar Arias ${ }^{5}$, Luis Such ${ }^{2,5}$, Javier Chorro ${ }^{3,5}$, José Millet ${ }^{1}$ \\ ${ }^{1}$ ITACA, Department Electronic Engineering, Universitat Politècnica de València, València, Spain \\ ${ }^{2}$ Departments of Physiology, Medicine ${ }^{3}$ and Physiotherapy ${ }^{4}$, Universitat de València, València, Spain \\ ${ }^{5}$ CiberCV, IIS Incliva-Cardiology Unit, Hospital Clínico Universitario, València, Spain
}

\begin{abstract}
Nowadays optical mapping (OM) is the primary method for imaging electrophysiologically relevant parameters from the outer surface of Langendorffperfused hearts. This technique has become essential for comprehensively understanding mechanisms of cardiac propagation during physiological activation, arrhythmia, and therapeutic antiarrhythmic interventions in translational hearts. Panoramic whole heart optical mapping systems, using either multiple cameras, plane mirrors or a combination of both, have been developed to overcome intrinsic visualization limitations to traditional single-sensor designs. However current panoramic OM systems are financially challenging for physiology and engineering research labs since require multiple sensors to completely map wide areas of the surface, which further results on overlapping regions and/or missing areas of interest due to the intrinsic shape of the heart. In this study, we propose and validate a new spatially resolved low-cost single-sensor configuration to simultaneously record atrial and ventricular function in relation to its anatomy without overlapping regions.
\end{abstract}

\section{Introduction}

Understanding comprehensively the mechanisms of spread of cardiac propagation at the organ level, and thus, characterize the distribution of transmembrane voltage throughout the heart, is one of the major objectives in cardiac electrophysiology $(1,2)$. The ability to record with high-resolution simultaneously multiple physiological parameters, as well as, to monitor large-scale phenomena such as fibrillation and/or defibrillation processes, has made $\mathrm{OM}$ a solid central technique to dynamically characterize function in relation to anatomy $(3,4)$.

This technique has enabled physiology researchers studying mechanisms of initiation and maintenance of life-threatening arrhythmias and the action of novel therapeutic interventions. Likewise, current therapeutic trends in clinical settings, either by using algorithms to accurately tracking and targeting arrhythmia drivers, and validation of predictive computational models used to test new therapeutic strategies in the clinical settings, have extensively benefited from developments in OM.

\subsection{Advances on panoramic OM}

Despite its widespread application in experimental and pre-clinical cardiac electrophysiology, OM of isolated hearts have been traditionally applied to record localized or constraint areas, missing critical information of the unknown dynamic processes occurring during discontinuous propagation in presence of arrhythmias. This is especially relevant during arrhythmia settings, for example, in presence of regional acute interventions or chronic substrate alterations, or simply when the interest is to characterize the spatiotemporal large-scale nonlinear dynamics of dominant driving mechanisms.

Nowadays, panoramic OM has become the primary method for imaging and mapping multiple electrophysiological parameters from the outer surface of the heart $(5,6)$. Yet, most alternatives obtain plane overlapping points-of-view with missing areas, and have been prohibitively expensive for research and teaching labs. Due to technological advancements, novel low-cost optimized approaches are gaining interest for imaging translational heart models (7). However, current alternatives are still limited regarding univocal continuity of the mapped heart surface including overlapping or missing regions. Here we propose a high-resolution single-sensor approach for simultaneously mapping atrial and ventricular outer surfaces of the heart.

The aim of this study was to compare and validate a new high-resolution and spatially-resolved low-cost 
single-sensor panoramic optical mapping configuration for simultaneous imaging and parametric mapping of atrial and ventricular activation during normal and abnormal activation.

\section{Methodology}

We designed an OM setup for studying whole heart electrophysiology using a single-sensor configuration in combination with a curved mirror with similar exponential curvature to that of the outer shape of the heart. After camera calibration and motion compensation, accurate reconstruction of optical transients and assessment of propagation under different conditions was done to evaluate reconstructed signal-to-noise ratios and overall mapping quality.

\subsection{Optical mapping system: Layout}

We devised a system layout integrated with the perfusion circuit comprising a single-view scientific camera (sensor element), a control system for led illumination, electrical stimulation and simultaneous sensing of physiological variables. A precision near ellipsoidal curved mirror (highly concentrated reflectance in the range $380 \mathrm{~nm}-800 \mathrm{~nm}$ ), with exponential profile and diameter to harbour small to large hearts was used to simultaneously obtain fluorescence from every point in the ventricular and atrial surfaces. A simplified schematic is shown in Figure 1. A four-point illumination array was placed surrounding the reflector element. The heart was perfused using a thin silicon-tube attached to a rigid body. Wired atrial electrodes were placed from a custom cannula unresponsive to light excitation.

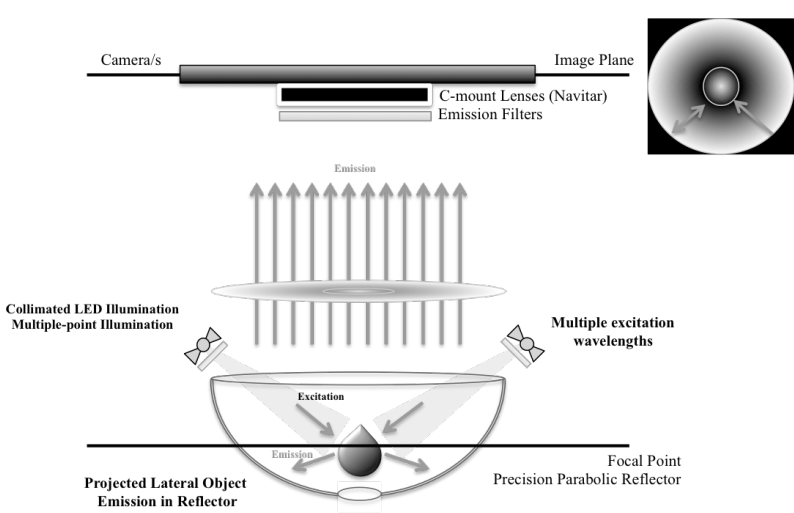

Figure 1. Proposed panoramic optical mapping system layout using a single-sensor configuration with a curved-parabolic reflector element.

Tyrode's perfusion liquid was collected from the bottom keeping the feedback circuit closed using a custom funnel-shaped cup with integrated electrodes to record a reference pseudo-ecg.

\subsection{Experimental models and protocol}

Six New Zealand White rabbits and one Large White pig were included in the study. Animal care and the experimental protocols used in the study complied with EU directive 2010/63 on the protection of animals used for scientific purposes, and were approved by the Institutional Animal Care and Use Committee of the University of Valencia (CEEA:A1404746730665). Rabbit hearts were excised through a medial sternal incision after anaesthetic pre-medication (ketamine $35 \mathrm{mg} / \mathrm{kg}$ and heparinization) and sodium-pentobarbitone $(100 \mathrm{mg} / \mathrm{Kg})$ overdose via injection in the left marginal ear vein. After removal, hearts placed in appropriately adjusted cold Tyrode's solution were connected into flow and pressure controlled Langendorff systems, respectively for rabbit and pig hearts. Hearts were stabilized and physiological variables monitored for 15-20 $\mathrm{min}$.

After stabilization we used 5-10uM blebbistatin to maintain the heart mechanics uncoupled during the experiment and reduce motion artifacts. High-resolution OM was performed using di-4-ANDBQPQ near-infrared dye. Illumination was collimated and passed through excitation filters fully exciting the whole myocardium. Fluorescence emission was appropriately collected and passed through a long-pass near infrared filter. Acquisition was performed using a EMCCD scientific camera (Evolve128, 1KHz, Photometrics, AZ, USA).

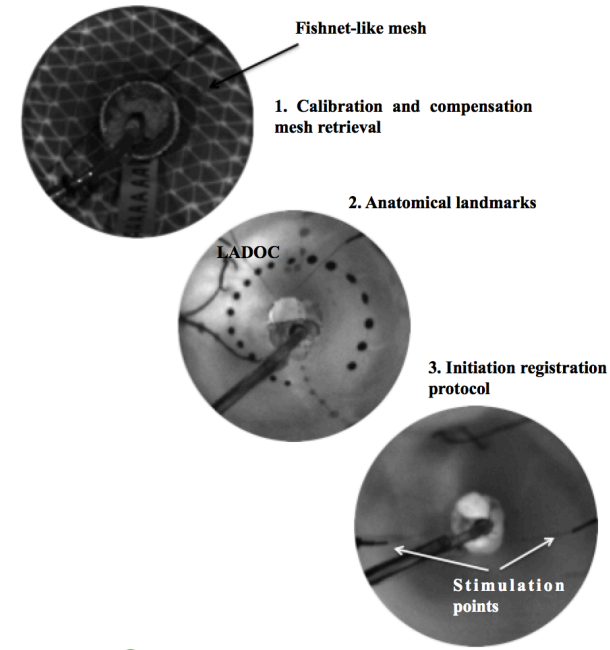

Figure 2. Fishnet-like pattern and anatomical landmarks unresponsive to light excitation used during calibration in Heart\#02-03.

Electrical signals and optical sequential images, were synchronized through a monostable-based triggering circuit. We characterized signal-to-noise ratios (SNR) of reconstructed action potentials (AP) during sinus rhythm and stimulation in specific anatomical locations. Hearts were stimulated to explore dynamic restitution properties and continued until ventricular fibrillation (VF) was 
induced with and without presence of a coronary occlusion in the LAD-artery (LADOC).

\subsection{Camera calibration and optical mapping reconstruction}

Initial camera calibration was done using a fishnetlike pattern to set the heart positioning and subsequent correspondence mapping. Nylon landmarks, as shown in Figure 2, unresponsive to light excitation were used as references to ensure accurate motion compensation using a block-matching cross-correlation strategy. Reconstructed optical maps were evaluated in terms of signal-to-noise ratios after normalization.

A
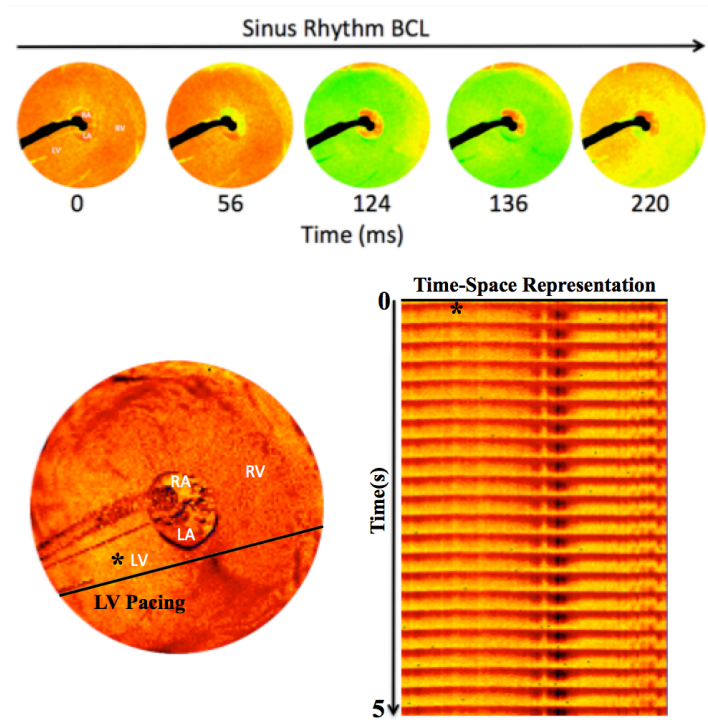

B

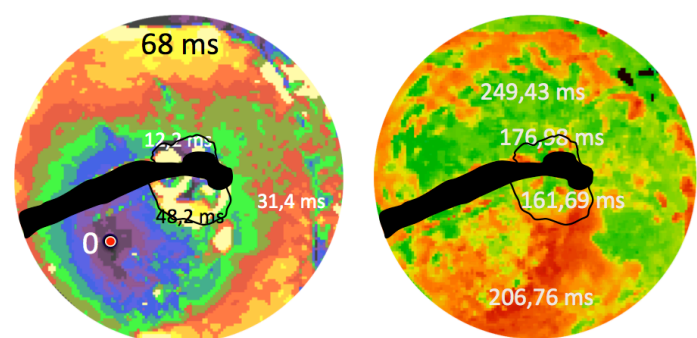

Figure 3. Activation process during sinus rhythm and during stimulation in one rabbit heart. A. Snapshots showing normalized fluorescence (F/Fo) during activation and recovery during sinus rhythm and time-space representation of paced activation. B. Activation map during ventricular pacing and $\mathrm{APD}_{80}$ map are shown.

\subsection{Quantitative post-processing}

Semi-automatic mask determination was applied using thresholding and connected component analyses for background removal. Labelling of different anatomical regions was done manually and refined using morphological post-processing. After reconstruction, fluorescence signals were detrended by baseline removal, normalized and filtered in time. We obtained parametric maps of the activation and repolarization spread propagation processes using first and second derivatives of the reconstructed signals. Action potential durations were obtained as durations from the activation to $80 \%$ of repolarization (Figure 3). Spectral dominant frequency mapping and phase mapping were used for fibrillatory conduction spatiotemporal assessment.

\section{Results}

Successful high-resolution OM showed high SNRs in atrial and ventricular surfaces (Figure 1, 28 $\pm 11 \mathrm{~dB}$ ). Figure 1, shows activation and recovery processes during sinus (A) and pacing (B), sequentially in the outer surface of each chamber of the heart. A time-space representation is shown during pacing illustrating propagation from the $\mathrm{LV}$ to the rest of the heart. Activation maps showed propagation throughout the heart surface. APD in selected locations highlight differences during fixed-point stimulation (APD80, ms) between left and right chambers in transmembrane potential transient durations.

Ventricular fibrillation, induced in the presence of a coronary occlusion, is illustrated in Figure 4, together with single pixel recordings in each chamber. Retrograde irregular activation is observed in the recorded atrial responses.
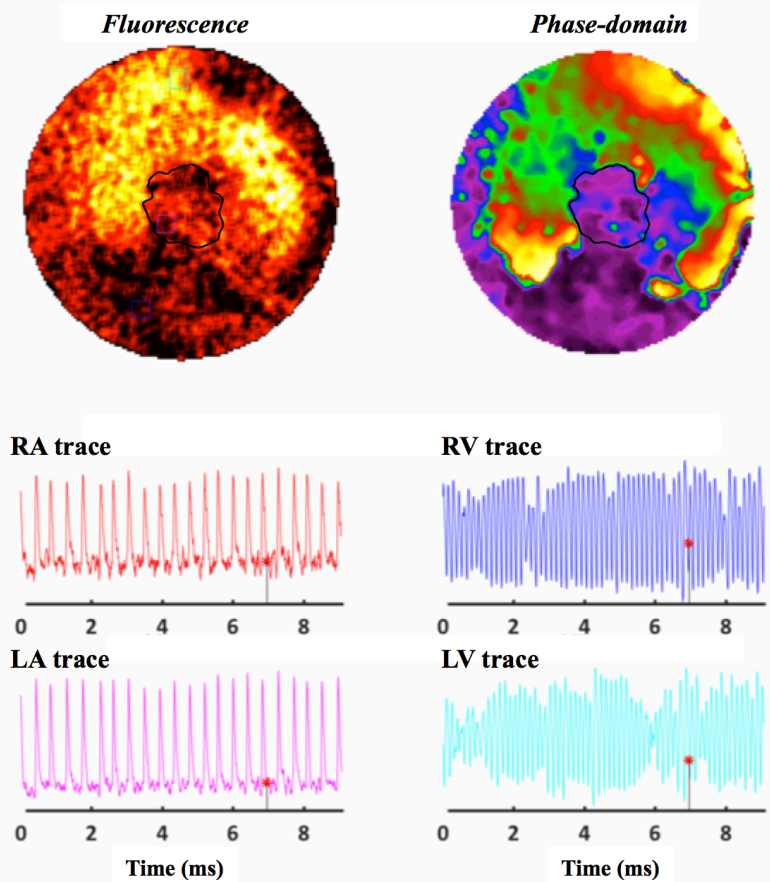

Figure 4. Snapshots of transmembrane voltage in the time domain (normalized fluorescence, top-left) and phase-domain (normalized Hilbert transform, top-right) during ventricular fibrillation in presence of a LAD coronary occlusion. Bottom. 
Single-pixel recordings from atrial and ventricular locations.

Characterization of the fibrillatory process in Heart\#04 is summarized in Figure 5. Wavebreak was observed near the coronary occlusion. Spatial profiles showed evident differences in dominant frequency and organization from the occlusion elsewhere, and between chambers.

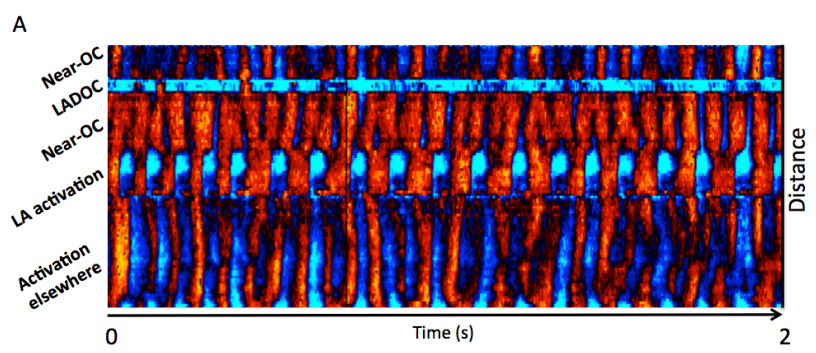

B

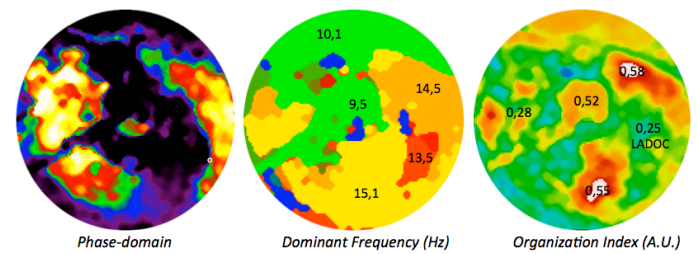

Figure 5. A. Time-space plot representation during fibrillatory activation in each region. B. Reconstructed phase-domain and DF mapping. Shown are high-resolution maps for OI and DF.

In this example, high activation and rotatory waves were observed surrounding the coronary occlusion. This configuration allowed for spatiotemporal identification of fibrillatory activation throughout the whole heart surface.

\section{Discussion and Conclusions}

In this study we devised a novel configuration to simultaneously map atrial and ventricular physiology without overlapping regions taking advantage of the heart shape. This configuration allowed us to reconstruct action potential transients and the spread propagation with highresolution. Even though the presented configuration may be useful as it is, it can be further optimized, and even lower its financial costs, as previously described (7).

Even though the preparation and calibration may be further optimized to improve reconstruction quality and/or reduce preparation times, it is clear that this configuration has proven useful to perform high-quality quantitative physiological studies and retrieve data simultaneously from whole-heart epicardial locations. Additionally, the presented configuration can be easily extended for multi-parametric mapping studies and/or combined with additional compatible techniques to obtain endocardial mapping and/or tank-based pseudo-ecg mapping to correlate activity from different layers. This information can be used to develop detailed mathematical models.
We validated a novel single-sensor configuration for parametric OM of cardiac propagation throughout the whole epicardial surface. Even though further optimization may be necessary to ensure homogeneous focusing, it may represent a new low-cost framework for physiology and engineering labs working in the fields of cardiac arrhythmias and whole-heart computational modeling.

\section{Acknowledgements}

This study has been supported by research grants GV/2015/019 (Generalitat Valenciana), FIS PI15/0074801408 and CB16/11/00486 (Instituto de Salud Carlos III).

\section{References}

1. Lin S, Wikswo JP. P ANORAMIC O PTICAL I MAGING OF E LECTRICAL P ROPAGATION IN I SOLATED H EART. 1999;4(2):200-7.

2. Efimov IR, Nikolski VP, Salama G. Optical imaging of the heart. Circ Res. 2004;95(1):21-33.

3. Herron TJ, Lee P, Jalife J. Optical Imaging of Voltage and Calcium in Cardiac Cells \&amp; Tissues. Circ Res [Internet]. 2012;110(4):609-23. Available from: http://circres.ahajournals.org/cgi/doi/10.1161/CIRCRE SAHA.111.247494

4. Boukens B, Efimov IR. a Century of Optocardiography. IEEE Rev Biomed Eng [Internet]. 2013;PP(99):1. Available from: http://ieeexplore.ieee.org/articleDetails.jsp?arnumber $=6$ 645408

5. Qu F, Ripplinger CM, Nikolski VP, Grimm C, Efimov IR. Three-dimensional panoramic imaging of cardiac arrhythmias in rabbit heart. J Biomed Opt [Internet]. 2007;12(4):44019. Available from: http://dx.doi.org/10.1117/1.2753748

6. Rogers J, Walcott G, Gladden JD, Melnick SB, Ideker RE, Kay MW. Epicardial wavefronts arise from widely distributed transient sources during ventricular fibrillation in the isolated swine heart. New J Physiol [Internet]. 2008;10(1):1-16. Available from: http://iopscience.iop.org/1367-2630/10/1/015004

7. Lee P, Calvo CJ, Alfonso-almazán JM, Quintanilla JG, Chorro FJ, Yan P, et al. Low-Cost Optical Mapping Systems for Panoramic Imaging of Complex Arrhythmias and Drug-Action in Translational Heart Models. Nat Publ Gr [Internet]. Nature Publishing Group; 2017;(February). Available from: http://dx.doi.org/10.1038/srep43217

Address for correspondence.

Conrado J. Calvo

Innovations on Bioengineering and Electrophysiology. Department of Electronic Engineering.

Universitat Politècnica de València.

Departament Fisiologia. Universitat de València.

conrado.calvo@uv.es 